\title{
Les protéines $G$ étendent leur pouvoir sur les canaux ioniques
}

Les protéines G (liant le GTP) constituent une famille de protéines homologues assurant le couplage à leurs effecteurs cellulaires de récepteurs aussi divers que la rhodopsine, les récepteurs adrénergiques ou les récepteurs aux odeurs. On pensait, il y a peu de temps encore, que ces protéines $G$ ne contrôlaient que des effecteurs enzymatiques produisant ou dégradant des seconds messagers. Or, depuis deux ans, on a découvert que ces protéines assuraient aussi le couplage direct entre des récepteurs membranaires et des canaux ioniques. Il apparaît, de plus, qu'un récepteur donné n'exécute pas une seule voie de couplage mais exécute tout un programme de transduction auquel coopèrent parfois plusieurs protéines $G$, chacune d'entre elles pouvant avoir plusieurs cibles dont l'activité est stimulée ou inhibée.

\section{Joèl Bockaert}

\section{RÉFÉRENCES}

1. Bockaert J. Les récepteurs membranaires. La Recherche 1986; 17 : 892-900.

2. Neer EJ, Clapham DE. Role of G protein structures in transmembrane signalling. Nature 1988 ; 333 : 129-34.

3. Homburger V, Brabet $P$, Audigier $Y$, Pantaloni C, Bockaert J, Rouot B. Immunological localisation of the GTP binding protein $G_{o}$ in different tissues of vertebrates and invertebrates. Mol Pharmacol 1987; 31: 313-9.

4. Brabet $P$, Dumuis A, Sebben M, Pantaloni C, Bockaert J, Homburger V. Immunocytochemical localisation of the guanine nucleotide binding protein $G_{0}$ in primary cultures of neuronal and glial cells. J Neurosci $1988 ; 8: 701-8$.

\section{ADRESSE}

J. Bockaert: directeur de recherche au Cnrs. Centre Cnrs-Inserm de pharmacologie-endocrinologie, rue de la Cardonille, 34094 Montpellier Cedex 2, France. 'évolution des êtres pluricellulaires est liée à la capacité qu'ont su développer leurs cellules à communiquer entre elles. Cette communication se fait par l'intermédiaire de messagers divers (hormones, neurotransmetteurs, facteurs de croissance) dont un grand nombre ne pénètre pas dans la cellule. Ces messagers sont reconnus par des récepteurs membranaires dont la stimulation induit une cascade d'événements biophysiques et biochimiques qui aboutiront à un changement d'une ou plusieurs propriétés de la cellule (contraction, division cellulaire, sécrétion, etc.) [1].

Une des découvertes les plus inattendues de ces cinq dernières années est que des récepteurs aussi différents que les récepteurs à la lumière (rhodopsine), les récepteurs adrénergiques et à beaucoup d'autres hormones ou neurotransmetteurs, ainsi que les récepteurs aux odeurs, agissent par l'intermédiaire de protéines membranaires de structures très homologues, liant le GTP et appelées protéines $G$ [2]. Une fois activée par le récepteur, la protéine $G$ qui lui est spécifiquement associée va modifier l'activité d'une ou plusieurs enzymes aussi diverses que l'adénylate cyclase, les phospholipases $\mathrm{C}$ ou $\mathrm{A}_{2}$ ou une phosphodiestérase activée par le GMPc. Jusqu'à ces trois dernières années, on ne connaissait que trois protéines $G: G_{s}, G_{i}$ - assurant respectivement le couplage positif et négatif de certains récepteurs avec l'adénylate cyclase - et la protéine $G_{\text {, }}$ aussi appelée transducine, assurant le couplage entre la rhodopsine et la phosphodiestérase dépendante de la GMPc des bâtonnets et des cônes rétiniens [2].

La découverte récente de nombreuses autres protéines $G$, comme $G_{o}$, une protéine $G$ très abondante dans les cellules nerveuses et certaines autres cellules excitables [3, 4], $G_{p}$ assurant le couplage entre le récepteur et la phospholipase $\mathrm{C}, \mathrm{G}_{\mathrm{ins}}$, intervenant dans l'action du récepteur de l'insuline, le fait qu'il existe des isoformes pour beaucoup d'entre elles (trois pour $G_{i}$, deux pour $G_{o}$, quatre pour $G_{s}$ ) ainsi que l'idée que beaucoup d'autres restent à découvrir, ont changé radicalement nos conceptions sur leur rôle.

Les protéines $G$ apparaissent à tort ou à raison comme le deus ex 
machina impliqué dans la régulation d'une vaste catégorie de processus de transduction, non seulement entre l'extérieur et l'intérieur de la cellule, mais aussi entre les différents compartiments membranaires de la cellule [5].

\section{Les protéines $G$}

Structure des protéines G. Les protéines $G$ assurant la transduction des récepteurs membranaires sont composées de trois sous-unités; la sousunité $\alpha$ (39 à $52 \mathrm{kDa}$ ), la sous-unité $\beta$ (35 et $36 \mathrm{kDa}$ ) et la sous-unité $\gamma$ (environ 5 à $8 \mathrm{kDa}$ ).

La sous-unité $\alpha$ est l'élément spécifique de chaque protéine $G$ et contient le site GTP. Il n'existe que deux sous-unités $\beta$ pour toutes les protéines $\mathrm{G}$ (35 et $36 \mathrm{kDa}$ ). Parmi les sous-unités $\gamma$ possibles, seule la sousunité $\gamma$ de $G_{1}$ a été purifiée et son ADNc cloné. C'est une protéine hydrophile, alors que les autres sousunités $\gamma$ sont hydrophobes. Les sousunités $\beta$ et $\gamma$ sont toujours étroitement associées et ne sont probablement jamais dissociées in vivo (figure 1). Seule la sous-unité $\gamma$ des protéines $G$ autres que $G_{t}$ pourrait être une protéine membranaire intrinsèque. Les sous-unités $\alpha$ et $\beta$ sont parfaitement hydrophiles. Malgré cela, il est vraisemblable que les sous-unités $\alpha$ peuvent adhérer à la membrane même une fois dissociées

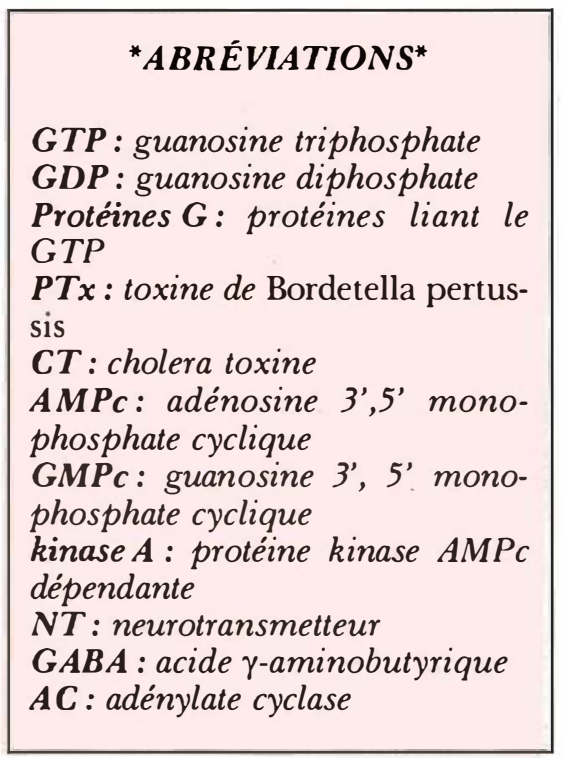

$\mathrm{m} / \mathrm{s} n^{\circ} 8$ vol. 5 , octobre 89

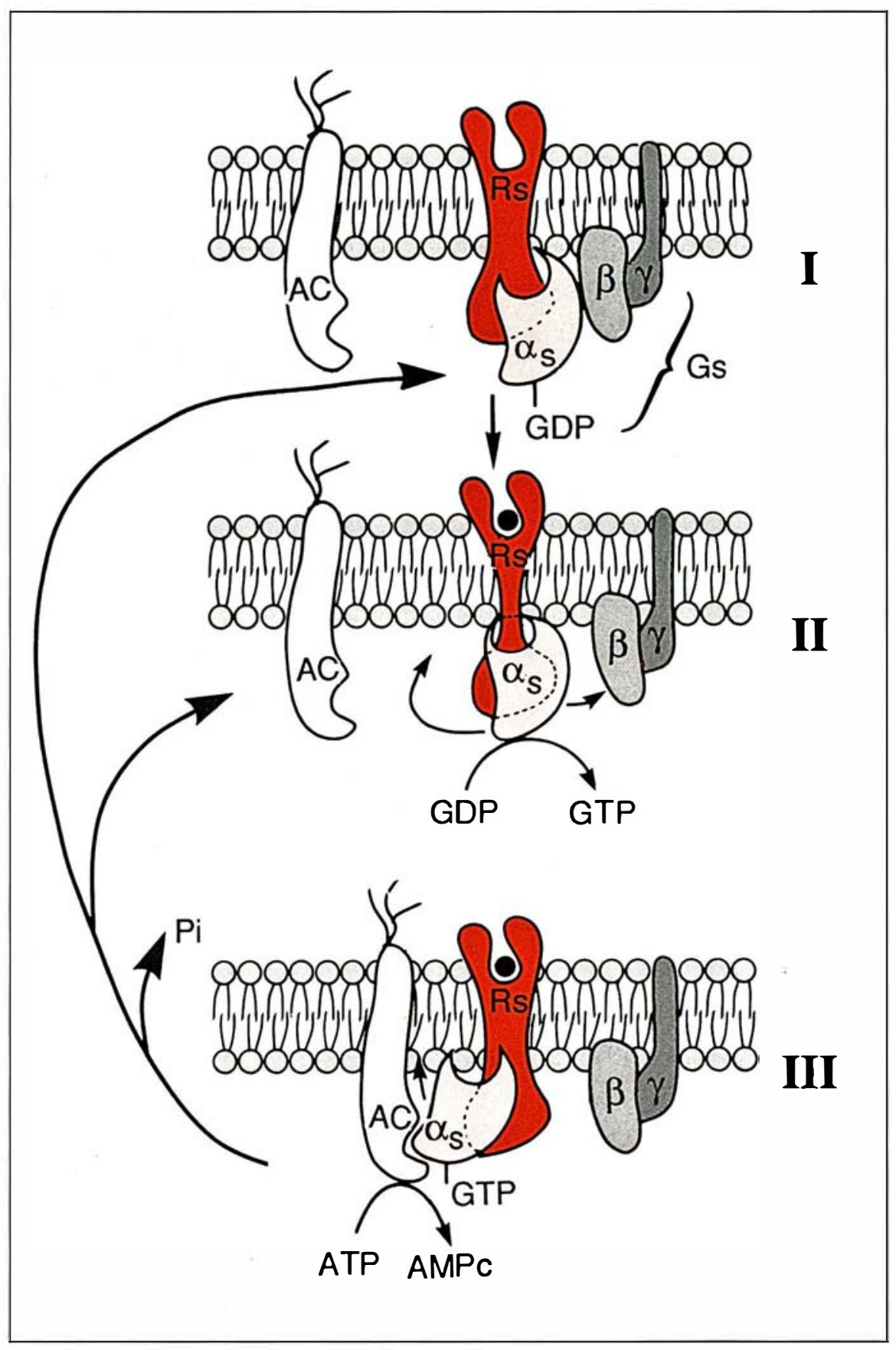

Figure 1. Mécanisme d'activation de l'adénylate cyclase (AC) par un récepteur stimulateur (Rs) par l'intermédiaire de la protéine liant le GTP (protéine G) de type $G_{s}$. I. En l'absence d'agonistes, la protéine $G_{s}$ est sous une forme trimérique $\alpha_{s} \beta \gamma$. Le site nucléotidique d' $\alpha_{s}$ est occupé par du GDP. Les sous-unités $\alpha$ et $\beta$ sont des molécules hydrophiles qui sont cependant fortement associées à la membrane. La sous-unité $\gamma$ est hydrophobe et intervient peut-être dans l'ancrage de $G_{s}$. La sous-unité $\gamma$ est représentée ici sous forme transmembranaire. Cela est une hypothèse. II n'est pas du tout certain que $G_{s}$ soit en interaction avec Rs en l'absence d'agonistes. II. En présence d'agoniste (•), le récepteur change de conformation, interagit avec $G_{s}$ et catalyse l'échange GDP $\rightarrow$ GTP sur $\alpha_{s}$ III. Une fois chargée de GTP, la sous-unité $\alpha_{s}$-GTP se dissocie de $\beta \gamma$ et va interagir avec l'AC pour l'activer. La réversibilité du système est assurée par I'hydrolyse du GTP. $G_{s}$ est une GTPase. On retourne alors à l'état ll, si le récepteur est encore occupé par l'agoniste, ou à l'état I, s'il ne l'est plus. Un récepteur occupé peut activer de nombreuses protéines $G$, ce qui assure une étape d'amplification. 


\section{RÉFÉRENCES}

5. Bourne HR. Do GTPases direct membrane traffic in secretion? Cell $1988 ; 53: 669-71$.

6. Fong $\mathrm{HKW}$, Yoshimoto KK, EversoleCire P, Simon MI. Identification of a GTP binding protein $\alpha$ subunit that lacks an apparent ADP-ribosylation site for pertussis toxin Proc Natl Acad Sci USA 1988 ; 85 : 3066-70.

7. Kozasa T, Itoh H, Tsukamoto T, Kaziro Y. Isolation and characterization of the human G $\alpha$ gene. Proc Natl Acad Sci USA 1988; 85 . 2081-5.

8. De Vos AM, Tong L, Milburn MV, et al Three dimensional structure of an oncogene protein : catalytic domain of human C-H ras p21. Science 1988 ; 239 : 888-93.

9. Touchot N, Chardin P, Tativian A. Four additional members of the ras gene superfamily isolated by oligonucleotide strategy Molecular cloning of YPT-related cDNA from a rat brain library. Proc Natl Acad Sci USA 1987 ; $84: 8210-4$.

10. Guy B, Kieng MP, Riviere Y, et al. HIV F/3' orf encodes a phosphorylated GTP binding protein resembling an oncogene product. Nature $1987 ; 330: 266-9$.

11. Reuter H. Calcium channel modulation by neurotransmitters, enzymes and drugs. Nature 1983 ; 301 : 569-74.

12. Hosey MM, Ladzunski M. Calcium channels : molecular pharmacology, structure and regulation. J Membr Biol 1988; 104 : 81-105.

13. DeterreP, Paupardin-Tritsch D Bockaert J, Gerschenfeld HM. cAMP mediated decrease in $\mathrm{K}^{+}$conductance evoked by serotonin and dopamine in the same neuron : a biochemical and physiological single cell study. Proc Natl Acad Sci USA 1982; 79 . 7934-8.

14. Goelet P, Castellucci VF, Schacher S Kandel F. The long and the short of long term memory: a molecular framework. Nature 1986 ; 322 : 419-22.

15. Hammond C, Paupardin-Trisch D, Nairn AC, Greengard P, Gerschenfeld HM Cholecystokinin induces a decrease in $\mathrm{Ca}^{++}$ current in snail neurons that appears to be mediated by protein kinase C. Nature 1987 ; $325: 809-911$.

16. FesenkoEE, KolesnikovSS et Lyubarky AL. Induction by cyclic GMP of a cationic conductance in plasma membrane of retinal rod outer segment. Nature $1985 ; 313$ : $310-3$.

17. Gold GH, Nakamura T. Cyclic nucleotide gated conductances : a new class of ion channels mediates visual and olfactory de $\beta \gamma$, une situation qui se produit lors de l'activation des protéines $\mathrm{G}$ par les récepteurs (figure 1).

Activation des protéines $\mathbf{G}$ par les récepteurs. Le modèle d'activation des protéines $G$ par les récepteurs membranaires est essentiellement fondé sur nos connaissances concernant l'activation de $\mathrm{G}_{\mathrm{s}}$ (figure 1).

Avant stimulation du récepteur par l'agoniste, $G_{\mathrm{s}}$ est sous forme d'un hétérotrimère $\alpha \beta \gamma$. Le site liant les guanylnucléotides est occupé par du GDP. Après activation par les agonistes, le récepteur change de conformation et interagit avec $G_{s}$. Il joue alors le rôle de catalyseur et stimule l'échange GDP-GTP. L'introduction du GTP sur $G_{s} \alpha$ entraîne la dissociation entre $G_{s} \alpha-G T P$ d'un côté et $\beta \gamma$ de l'autre. $G_{s} \alpha$-GTP active alors l'adénylate cyclase. L'activation de l'adénylate cyclase cesse lors de l'hydrolyse du GTP en GDP. Le temps moyen d'occupation d'un récepteur par l'agoniste permet à celui-ci d'activer de nombreuses protéines G. Cela assure une étape d'amplification.

Les protéines G, site d'action de toxines. Les toxines bactériennes du Vibrio cholerae (CT) et de Bordetella pertussis (PTx) peuvent modifier de façon covalente les sous-unités $\alpha$ de certaines protéines $\mathrm{G}$. Ces toxines sont des enzymes de type ADP-ribosyltransférase qui catalysent le transfert de l'ADP-ribose du $\mathrm{NAD}^{+}$sur la sous-unité $\alpha$. Les protéines $G$ peuvent être divisées en quatre groupes, celles qui sont uniquement des substrats de la CT $\left(G_{s}\right)$, celles qui sont uniquement des substrats de la PTx $\left(G_{0}\right.$ et $\left.G_{i}\right)$, celles qui sont des substrats des deux $\left(G_{1}\right)$ et celles qui sont des substrats d'aucune $\left(G_{\iota}\right)[6]$. La CT active $G_{s}$, alors que la PTx inhibe le couplage entre les protéines $G$ et les récepteurs qui leur sont associés. La PTx est donc un bon outil pour démontrer le couplage entre un récepteur et une protéine $\mathrm{G}$.

Les protéines $\mathbf{G}$, une famille nombreuse. La purification des protéines $G$ et le clonage de leurs ADNc ont révélé l'existence d'une variété de sous-unités $\alpha$. Il y a quatre formes de $\mathrm{G}_{\mathrm{s}} \alpha$ obtenues à partir de l'épissage différentiel d'un seul messager précurseur [7]. Il y a au moins trois types de sous-unités $\alpha$ de $G_{i}$, appelées $G_{i} \alpha_{1}$ $(41 \mathrm{kDa}), G_{i} \alpha_{2}(40 \mathrm{kDa}), \mathrm{G}_{\mathrm{i}} \alpha_{3}$ (4l kDa), deux types de $\mathrm{G}_{0} \alpha(39 \mathrm{kDa})$ (travaux non publiés du laboratoire), deux types de $G_{t} \alpha$, l'une présente dans les bâtonnets, l'autre dans les cônes [2]. D'autres sous-unités $\alpha$ sont encore à découvrir, notamment $G_{p}$ assurant le couplage récepteur-phospholipase C.

En dehors des protéines $G$ hétérotrimériques, il existe d'autres familles de protéines $\mathrm{G}$ dont l'homologie avec les premières se situe surtout au niveau du site de liaison du GTP [8]. Ces familles comprennent les protéines de type Ras, Rho, Ral, Rab des cellules eucaryotes [9], les protéines YPTl et SEC4 [5] de la levure, la protéine F du virus HIV [10], les facteurs d'élongation et d'initiation des protéines. Leurs rôles pourraient être divers, transduction des effets des facteurs de croissance (Ras), transport vectoriel de substrats (facteurs d'initiation et d'élongation) ou de vésicules membranaires (YPTl et SEC4) [5].

\section{Protéines G et canaux ioniques}

Contrôle indirect des canaux ioniques par les protéines $\mathbf{G}$. Les canaux ioniques entrent classiquement dans deux catégories. Dans la première, le canal ionique et le site de reconnaissance du neurotransmetteur sont portés par la même molécule (figure $2 A$ ). Cette catégorie comprend les récepteurs nicotiniques de l'acétylcholine, les récepteurs GABA A de l'acide $\gamma$-aminobutyrique (GABA), les récepteurs à la glycine. L'interaction du neurotransmetteur avec son site de liaison entraîne un changement de conformation qui laisse passer des ions dans le canal. Dans la deuxième catégorie, le canal est situé sur une molécule distincte de celle que reconnaît le neurotransmetteur. Deux types de couplage existent. Le premier est maintenant bien établi (figure $2 \boldsymbol{B}$ ). Le récepteur, une fois activé, interagit avec une protéine $G$ qui ellemême active une enzyme qui produit un second messager. Les protéines $G$ les plus souvent rencontrées dans ce type de couplage sont $G_{s}$ (qui stimule l'adénylate cyclase) et $G_{p}$ (qui 


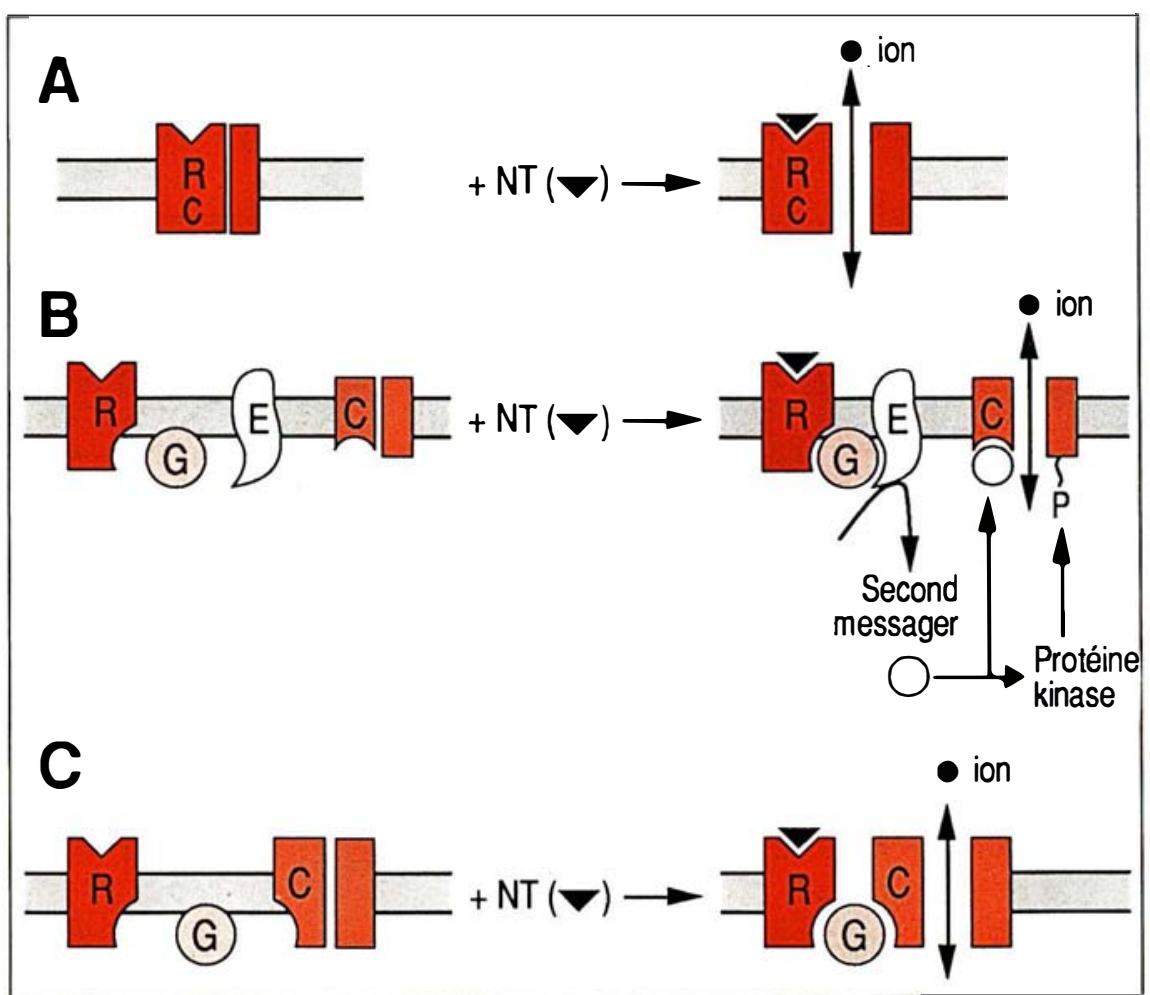

Figure 2. Les différents mécanismes de contrôle des canaux ioniques par des récepteurs membranaires. A. Le récepteur $(R)$ et le canal ionique $(C)$ font partie de la même molécule. Le neurotransmetteur (NT) une fois fixé sur cette molécule ouvre le canal ionique. Les récepteurs de ce type les mieux connus sont les récepteurs nicotiniques de l'acétylcholine, les récepteurs NMDA du glutamate et les récepteurs GABAA du GABA. B. Le récepteur active (ou inhibe) une enzyme (E) qui va produire un second messager. Celui-ci active une protéine kinase qui, en phosphorylant le canal ionique (la protéine kinase peut, au contraire, activer des phosphatases qui déphosphorylent le canal ionique), modifie son activité. Le cas le mieux connu de ce type de transduction est le contrôle des canaux $\mathrm{Ca}^{++}$ cardiaques par la noradrénaline (11). Le second messager peut aussi activer ou inhiber directement le canal ionique. Le cas le mieux connu de ce type de transduction est la régulation du canal $\mathrm{Na}^{+}$des bâtonnets rétiniens par le GMPc [16]. C. Le récepteur est directement couplé, par l'intermédiaire d'une protéine $G$, au canal ionique. Le cas le mieux connu de ce type de transduction est le contrôle des canaux $\mathrm{K}^{+}$par le récepteur muscarinique cardiaque de l'acétylcholine [20].

stimule la phospholipase C) assurant la production respectivement de l'AMPc et d'inositol triphosphate $\left(\mathrm{IP}_{3}\right) /$ diacylglycérol (DAG). Parmi les autres seconds messagers impliqués dans le contrôle de l'activité des canaux ioniques, citons le GMPc et l'acide arachidonique et ses dérivés. Comment les seconds messagers contrôlent-ils l'activité des canaux ? Les mécanismes classiques sont illustrés (figure $2 B$ ). Le second messager peut activer une protéine kinase qui phosphorylera directement le canal. L'exemple le mieux connu de ce type $\mathrm{m} / \mathrm{s} n^{\circ} 8$ vol. 5 , octobre 89 line. On sait, d'autre part, que les canaux $\mathrm{Ca}^{++}$sont phosphorylés par diverses kinases dont la kinase A [12]. Des études utilisant la méthode de patch-clamp (voir $\mathrm{m} / \mathrm{s} n^{\circ} 9$, vol. 3, p. 538) en configuration cell-attached (figure 4A) ont bien montré que le signal nécessaire à l'ouverture du canal $\mathrm{Ca}^{++}$est la dépolarisation brève (canal voltage-dépendant), mais que la noradrénaline ou l'AMPc augmente la probabilité d'ouverture du canal pour une dépolarisation donnée. Parmi les autres canaux dont l'activité est modulée par l'AMPc, citons un canal $\mathrm{K}^{+}$impliqué dans la libération des neuromédiateurs de certains neurones de mollusques [13] et qui jouent un rôle important dans les phénomènes de mémoire élémentaires [14]. Parmi les canaux inactivés et le DAG (qui active une kinase $\mathrm{C}$ ), citons les canaux $\mathrm{Ca}^{++}$-voltagedépendants [15] mais aussi certains canaux $\mathrm{K}^{+}$.

Le deuxième mécanisme par lequel un second messager peut contrôler l'activité d'un canal a été découvert très récemment par Fesenko [16]. Il s'agit d'une interaction directe du second messager avec le canal (figure 2B). Fesenko a pu montrer un effet direct du GMPc sur l'activité du canal $\mathrm{Na}^{+}$des bâtonnets rétiniens. Pour cela, il a utilisé la version insideout de la technique de patch-clamp qui permet d'arracher un petit fragment de membrane qui reste accolé à la pipette ( $1 \mu \mathrm{m}$ de diamètre) (figure 4A). Ce fragment de membrane contient un ou plusieurs canaux dont on peut mesurer l'activité. Elle permet de manipuler la composition des milieux baignant les faces cytoplasmique (bain dans lequel est placé la pipette) et extracellulaire (liquide de la pipette). Fesenko a montré que le GMPc, mis dans le bain, ouvre les canaux $\mathrm{Na}^{+}$ du fragment de membrane accolé à la pipette [16]. Cela exclut $a$ priori un effet indirect et l'intervention d'une kinase dépendante de la GMPc. De même, les can a ux $\mathrm{Na}^{+} \mathrm{de} \mathrm{la}$ muqueuse olfactive sont directement activés par l'AMPc et le GMPc [17]. Il existe aussi des arguments en faveur d'un effet direct de l'I $\mathrm{P}_{3}$ sur des canaux $\mathrm{Ca}^{++}[18]$.

Contrôle direct des canaux ioniques par les protéines $\mathbf{G}$. Le deuxième 


\section{RÉFÉRENCES}

18. Kuno M, Gorony J Weyand C Gardner $\mathrm{P}$. Single channel and whole cell recordings of mitogen regulated inward currents in human cloned helper lymphocytes. Nature 1986 ; 323 : $269-73$.

19. Pfaffinger PJ, Martin JM, Hunter DD, Nathanson NN, Hille B. GTP binding proteins couple cardiac muscarinic receptors to a K ${ }^{+}$channel. Nature $1985 ; 317$ : 536-8.

20. Yatani A, Codina J, Brown AM, Birnbaumer $\mathrm{L}$. Direct activation of mammalian atrial muscarinic potassium channels by GTP regulatory protein $G_{k}$. Science $1987 ; 235$ : 207-11.

21. Kim D, Lewis DL, Graziadei L, Neer EJ, Bar-Sagi D, Clapham DE. G protein $\beta \gamma$ subunits activate the cardiac muscarinic $\mathrm{K}^{+}$channel via phospholipase $\mathrm{A}_{2}$. Nature $1989 ; 337$ : 557-60.

22. Yatani A, Mattera R, Codina J, et al. The $\mathrm{G}$ protein gated atrial $\mathrm{K}^{+}$channel is stimulated by three distinct $G_{i} a$ subunits. Nature $1988 ; 336: 680-2$.

23. Yatani A, Imoto $Y$, Codina J, Hamilton SL, Brown AM, Birnbaumer L. The stimulatory $G$ protein of adenylyl cyclase, $G_{s}$ also stimulates dihydropyridine-sensitive $\mathrm{Ca}^{++}$channels. J Biol Chem $1988 ; 263: 9887$. 95 .

24. Harris-Warrick RM, Hammond C, Paupardin-Tritsch D, et al. An $\alpha-40$ subunit of a GTP binding protein immunologically related to $G_{0}$ mediates a dopamine induced decrease of $\mathrm{Ca}^{++}$current in snail neurons. Neuron $1988 ; 1$ : 27-32.

25. Hescheler J, Rosenthal W, Trautwein W, SchultzG. The GTP binding protein, $G_{o}$ regulates neuronal calcium channels. Nature 1987 ; 325 : 445-7.

26. Van Dongen AMJ, Codina J, Olate J, et al. Newly identified brain potassium channels gated by the guanine nucleotide binding protein $\mathrm{G}_{\mathrm{o}}$. Science $1988 ; 242$ : 1433-7.

27. Journot $L$, Homburger V, Pantaloni C, Priam M, Bockaert J, Enjalbert A. An islet activating sensitive $G$ protein is involved in dopamine inhibition of angiotensin and TRH-stimulated inositol phosphate production in anterior pituitary cells. J Biol Chem 1987 ; 262 : 15106-10.

28. FarfelZ, Brothers VM, Brickman AS, Conte F, Neer R, Bourne HR. Pseudohypoparathyroidism patients : evidence that distinct receptor cyclase coupling proteins mediate stimulation and inhibition of adenylate cyclase. Proc Natl Acad Sci USA 1981; 78 : 3098-102.

29. Vallar L, Spada A, Giannattasio G. Altered $G_{s}$ and adenylate cyclase activity in human $\mathrm{GH}$-secreting pituitary adenomas.
A
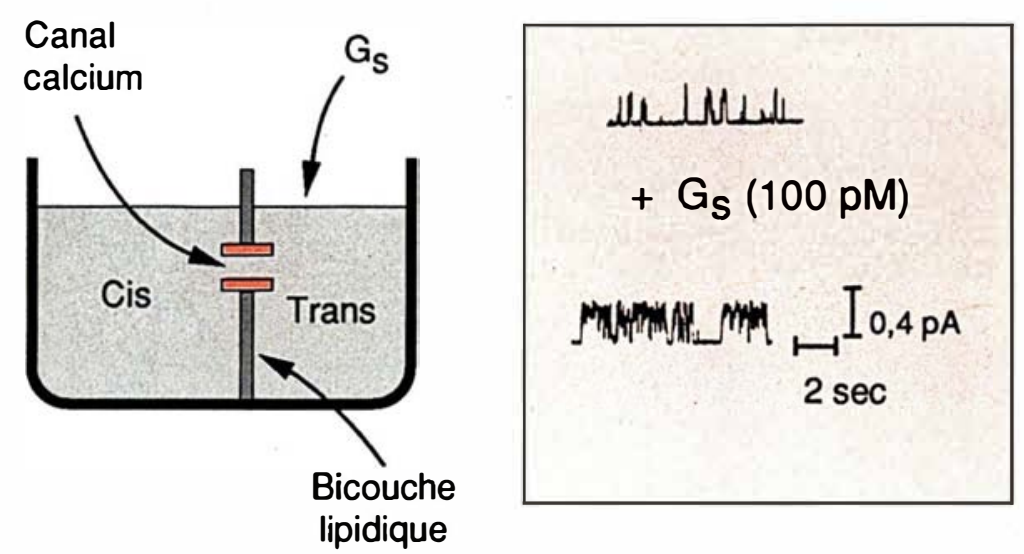

B

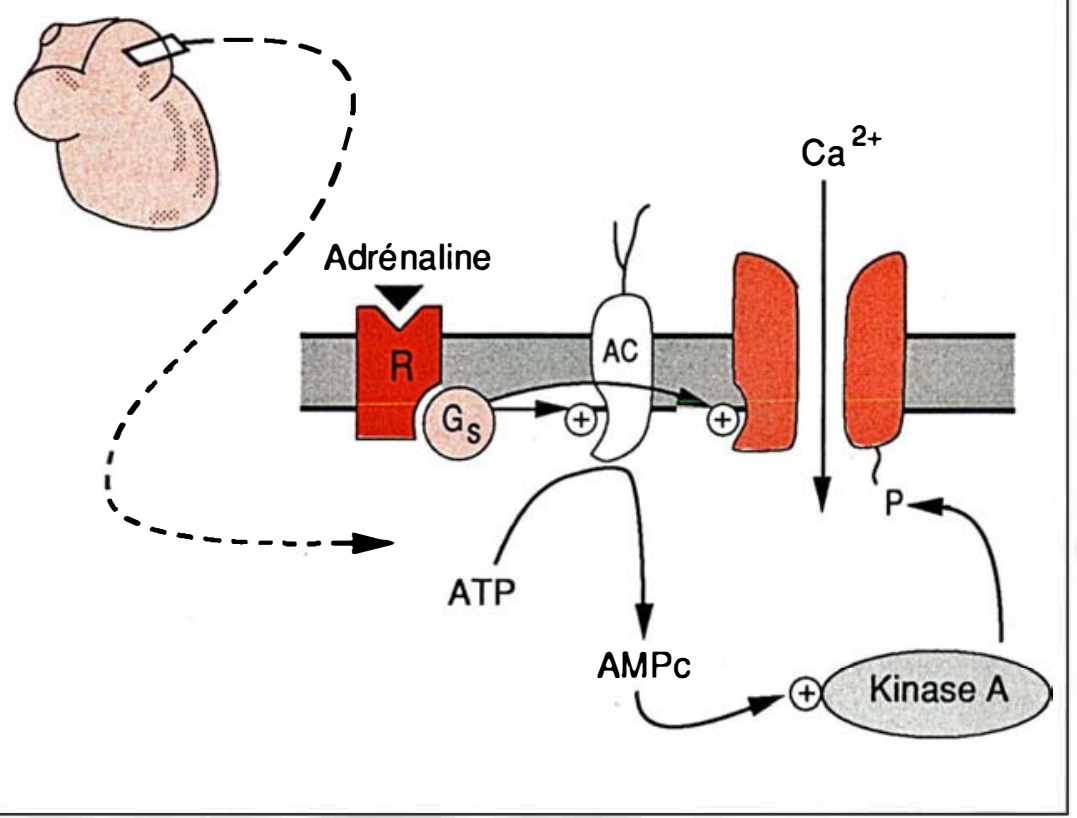

Figure 3. Contrôle des canaux $\mathrm{Ca}^{++}$-voltage-dépendants par la protéine $\boldsymbol{G}_{\mathrm{s}}$ et la protéine kinase A. A. Une bicouche lipidique (phosphatidyléthanolamine et phosphatidylsérine) est insérée entre deux compartiments cis et trans. Des vésicules membranaires contenant des canaux $\mathrm{Ca}^{++}$-voltage-dépendants sont introduites dans le compartiment cis. De façon aléatoire, des canaux $\mathrm{Ca}^{++}$de ces vésicules fusionnent avec la bicouche. On peut alors enregistrer l'activité de canaux élémentaires. Lorsque la protéine $G_{s}(100 \mathrm{pM}$ ) activée par le GTP- $\gamma$-S (afin d'obtenir la dissociation entre $G_{s} \alpha-G T P-\gamma-S$ et $\beta \gamma$ ) est introduite dans le compartiment trans, la fréquence $d^{\prime}$ ouverture des canaux $\mathrm{Ca}^{++}$-voltage-dépendants augmente (d'après [23]). B. Au niveau du cœur, l'augmentation de l'activité des canaux $\mathrm{Ca}^{++}$ voltage-dépendants par la noradrénaline pourrait se faire par deux voies. L'une est maintenant classique. Le récepteur $\beta$-adrénergique active l'adényl cyclase par l'intermédiaire de $G_{g}$ comme nous l'avons décrit dans la figure 1. L'AMPc active une protéine kinase $A$. La phosphorylation, par la protéine kinase $A$, du canal $\mathrm{Ca}^{++}$ augmente son activité. L'autre voie, moins classique, est suggérée par l'expérience décrite en $A$. Le récepteur $\beta$-adrénergique active la protéine $G_{s}$ et celle-ci va directement activer le canal $\mathrm{Ca}^{++}$-voltage-dépendant. 


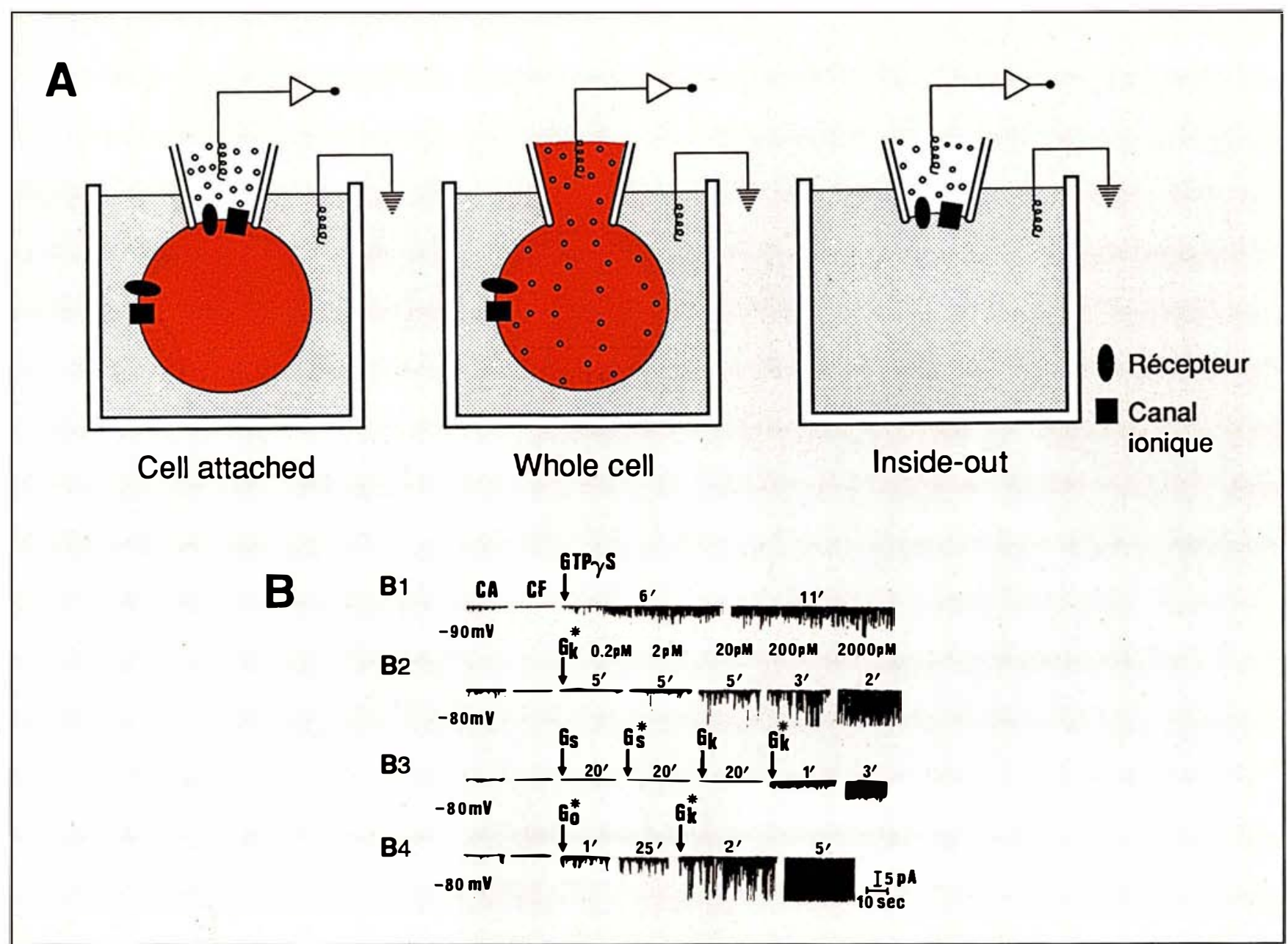

Figure 4. Les différentes configurations utilisées dans la méthode de patch clamp pour étudier l'interaction des récepteurs avec les canaux ioniques (A). Contrôle des canaux $K^{+}$cardiaques par les protéines $G$ étudié dans la configuration "inside out " (B). A. Cell attached : la pipette de patch est déposée sur la cellule et la membrane cellulaire aspirée légèrement à l'intérieur de la pipette. On enregistre l'activité des canaux isolés dans ce fragment de membrane. Whole cell : la rupture de la membrane sous la pipette de patch de la configuration précédente conduit à la configuration « whole cell $\star$. II y a alors communication entre le milieu de la pipette et celui de la cellule dont on peut donc modifier la composition. On enregistre alors l'ensemble des courants de la cellule. Inside out : en soulevant la pipette en configuration * cell-attached , on peut arracher un fragment de membrane dont la surface interne est alors mise en contact avec le milieu ionique du bain. On peut avoir, dans ce fragment de membrane, des canaux seuls ou avec des récepteurs. B. Les canaux $K^{+}$de l'oreillette cardiaque sont étudiés dans la configuration *inside-out $\%$. On rajoute alors dans le bain du GTP- $\gamma-S(B 1)$, différentes concentrations de protéine $G_{k}(B 2), G_{s}(B 3)$ ou $G_{o}(B 4)$. Toutes ces protéines sont activées par le GTP- $\gamma$-S (astérisque) (d'après [20]).

mécanisme de contrôle des canaux ioniques par les protéines $\mathrm{G}$ est direct. Il a été tout récemment découvert (figure $2 C$ ). La protéine $\mathbf{G}$, une fois activée par le récepteur, interagit directement avec le canal ionique, soit pour l'ouvrir; soit pour le fermer. Ici encore, le couur a servi de modèle. La liaison de l'acétylcholine au récepteur muscarinique cardiaque active un canal $\mathrm{K}^{+}$dit canal de rectification qui inhibe l'activité pacemaker cardiaque [19]. Lorsque l'acti-' vité des canaux $\mathrm{K}^{+}$de ces cellules est $\mathrm{m} / \mathrm{s} n^{\circ} 8$ vol. 5 , octobre 89 mesurée sous la pipette de patch dans la configuration cell-attached (figure 4A), l'acétylcholine n'a aucune action lorsqu'elle est appliquée à l'extérieur de la pipette, excluant un contrôle par production de seconds messagers [19]. Seule une application à l'intérieur de la pipette est efficace. Cela pouvait suggérer que ce récepteur est un récepteurcanal. Cependant, la mesure de l'activité de ces canaux selon la configuration whole cell (figure $4 A$ ), dans laquelle le milieu intracellulaire de la cellule communique avec l'intérieur de la pipette, montre que si cette pipette ne contient pas de GTP, l'acétylcholine mise cette fois à l'extérieur de la cellule n'est pas capable d'activer les canaux $\mathrm{K}^{+}$[19]. Le GTP$\gamma-S$, un analogue non hydrolysable du GTP, mis dans la pipette est, lui, capable d'activer irréversiblement le canal $\mathrm{K}^{+}$. De plus, l'incubation des cellules cardiaques avec la PTx inhibe l'effet de l'acétylcholine [19]. Une protéine $\mathbf{G}$ est donc impliquée dans le couplage « récepteur musca- 


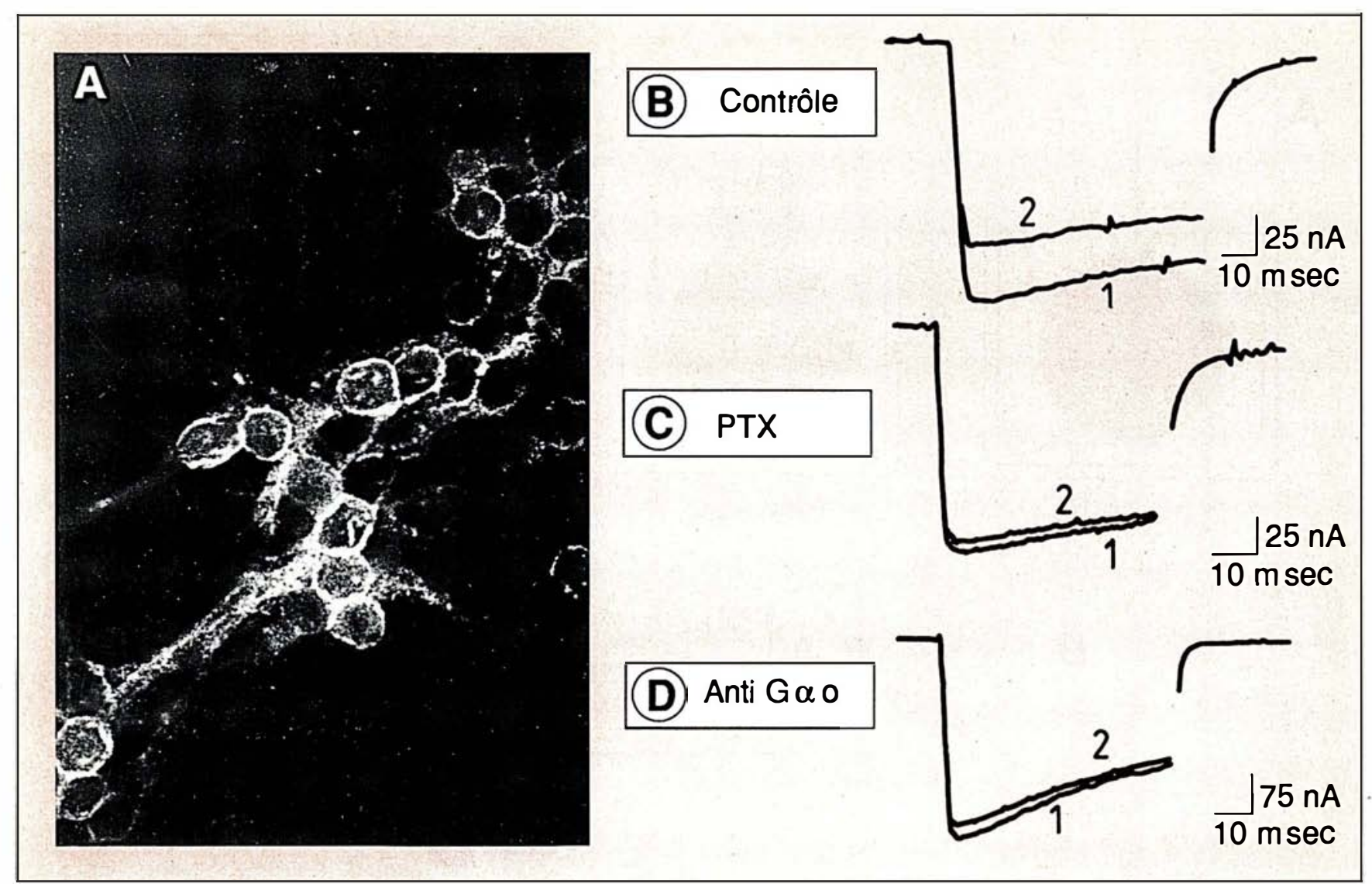

Figure 5. La protéine $G_{o}$ des cellules excitables inhibe des canaux Ca ${ }^{++}$-voltage-dépendants. A. La protéine $G_{0}$ est détectée en immunofluorescence dans des neurones (granules) du cervelet (X 620). La protéine $G_{o}$ est particulièrement localisée au niveau des membranes et essentiellement au niveau des contacts cellulaires (d'après [4]). B. Courant passant par les canaux $\mathrm{Ca}^{++}$-voltage-dépendants des neurones d'escargot [1] et sa réduction par la dopamine [2]. C. L'injection de la toxine de Bordetella pertussis $(P T X)$, qui inactive les protéines $G_{o}$ et $G_{i}$ supprime, l'effet de la dopamine $[1,2]$. D. L'injection de l'anticorps anti-G $G_{o} \alpha$ supprime aussi l'effet de la dopamine (d'après [24]).

rinique-canal $\mathrm{K}^{+}$» du cœur sans qu'il soit nécessaire de produire un second messager. La démonstration du couplage direct entre ces trois protéines est venue de l'utilisation de la technique inside-out (figure 4A). Sur des fragments de membrane isolés du milieu cytoplasmique, les équipes de A.M.Brown et L. Birnbaumer ont bien montré que: (1) le GTP- $\boldsymbol{\gamma}$-S active les canaux $\mathrm{K}^{+}$; (2) de très faibles concentrations (picomolaires) d'une protéine $G$, qu'ils ont appelé $G_{k}$, et qui se révèle être $G_{i 3}$, active les canaux $\mathrm{K}^{+}$(figure 4B) [20].

La protéine $\mathrm{G}$ utilisée est activée par le GTP- $\gamma-S$ et se. trouve donc sous

plus $\beta \gamma$. Des concentrations mille fois plus fortes de $G_{\mathrm{s}}$ activée sont totalement inefficaces (figure $4 B$ ). $G_{0}$ activée peut ouvrir ces canaux $\mathrm{K}^{+}$ cardiaques, mais avec une activité 100 fois moindre que $G_{k}$. Notons qu'il est impossible d'exclure que dans un patch en configuration inside-out une ou plusieurs autres protéines intermédiaires puissent être mises en jeu entre la protéine $G$ et le canal $\mathrm{K}^{+}$. La possibilité d'interactions complexes entre protéines au sein même d'un patch inside-out a été révélée très récemment. En effet, des concentrations nanomolaires de sous-unités $\beta \gamma$ peuvent aussi activer ce canal dans un patch en insideout [2]. L'effet de $\beta \gamma$ sur le canal $\mathrm{K}^{+}$ est complexe et semble se faire par l'intermédiaire de la production d'acide arachidonique et ses dérivés au sein même du patch [21]. L'acétylcholine n'emprunte cependant pas cette voie et son action se fait bien par l'intermédiaire de la sous-unité $\alpha$ de $G_{k}[21]$.

Le couplage au sein même de la membrane entre le récepteur et le canal, par l'intermédiaire d'une protéine $G$ et sans synthèse de seconds messagers, pourrait expliquer pourquoi la stimulation vagale produit des effets plus rapides sur le rythme cardiaque que la stimulation sympathique. Des expériences récentes ont montré qu'in vitro, non seulement $G_{k}\left(G_{i 3}\right)$ mais aussi $G_{i 1}$ et $G_{i 2}$ peuvent 
activer le canal $\mathrm{K}^{+}$[22]. Ce résultat indique qu'une (ou plusieurs) sousunité(s) $\mathrm{G}_{i} \alpha$ est (sont) capable(s) à la fois d'activer le canal $\mathrm{K}^{+}$de rectification cardiaque, mais aussi d'inhiber l'adénylate cyclase. Cette notion nouvelle, selon laquelle une seule protéine $G$ pourrait activer et/ou inhiber plusieurs effecteurs, commence à émerger. En effet, il a été aussi récemment démontré que $G_{s}$ est capable non seulement d'activer l'adénylate cyclase, mais aussi d'augmenter directement l'activité de canaux $\mathrm{Ca}^{++}$-voltage-dépendants incorporés dans une bicouche lipidique (figure $3 A$ ) [23]. Si les résultats de cette expérience pouvaient être extrapolés à ce qui se passe dans la cellule cardiaque, on aurait un double contrôle du canal $\mathrm{Ca}^{++}$-voltagedépendant, le premier indirect par l'AMPc via la kinase $\mathrm{A}$, le deuxième direct via $\mathrm{G}_{\mathrm{s}}$ (figure $3 B$ ). L'intérêt physiologique d'un double contrôle des canaux $\mathrm{Ca}^{++}$par $\mathrm{G}_{\mathrm{s}}$ n'est pas connu.

Nous venons de voir qu'une protéine $G$ peut contrôler deux effecteurs différents, une enzyme et un canal ionique. La protéine $G_{o}$ semble pouvoir contrôler deux canaux différents. En effet nous avons montré[24], en collaboration avec l'équipe de H. Gerschenfeld, qu'une protéine $G$ reconnue par un anticorps spécifique dirigé contre $G_{0} \alpha$ couple de façon négative les récepteurs dopaminergiques de neurones d'escargots aux canaux $\mathrm{Ca}^{++}$-voltage-dépendants. La PTx bloque ce couplage ainsi que l'injection d'anticorps anti- $G_{0} \alpha$ dans le neurone (figure 5) [24]. Une inhibition des canaux $\mathrm{Ca}^{++}$par $\mathrm{G}_{\mathrm{o}}$ a été également démontrée dans des neuroblastomes [26]. Plus récemment, il a été rapporté que $G_{o}$ était aussi capable d'activer certains canaux $\mathrm{K}^{+}$des neurones de l'hippocampe (différents de ceux des cellules cardiaques) [25]. $G_{o}$ pourrait donc activer des canaux $\mathrm{K}^{+}$ et inhiber des canaux $\mathrm{Ca}^{++}$. Cela est intéressant car une catégorie de neurotransmetteurs inhibiteurs comprenant les récepteurs dopaminergiques $\mathrm{D}_{2}$, les récepteurs des enképhalines, les récepteurs de la sérotonine de type $5-\mathrm{HT}_{1 \mathrm{~A}}$, les récepteurs $\mathrm{GABA} \mathrm{B}$, les récepteurs de l'adénosine de type $A_{1}$ sont connus pour activer des canaux $\mathrm{K}^{+}$et inhiber des canaux $\mathrm{Ca}^{++}$.

$\mathrm{m} / \mathrm{s} n^{\circ} 8$ vol. 5 , octobre 89

\section{Conclusion}

La découverte récente que les protéines $G$ contrôlent non seulement la production de seconds messagers, mais aussi, directement, l'activité de canaux ioniques, souligne bien le principe général d'action de ces protéines : la liaison du GTP catalysée par des protéines chargées de reconnaître un message, est utilisée pour produire un changement de conformation des protéines $G$. L'évolution a utilisé ce changement de conformation d'une manière créative pour contrôler l'activité d'effecteurs variés. Il est possible que des effecteurs autres que des enzymes ou des canaux ioniques soient contrôlés par les protéines $G$. On peut penser aux protéines du cytosquelette, mais aussi aux séquences régulatrices de gènes. L'autre idée importante qui émerge est que les mécanismes de transduction d'un récepteur sont multiples. Le récepteur dopaminergique $\mathrm{D}_{2}$ par l'intermédiaire d'une ou plusieurs protéines $G$, toutes sensibles à la PTx, inhibe l'adénylate cyclase, active un canal $\mathrm{K}^{+}$, inhibe certainement un canal $\mathrm{Ca}^{++}$, inhibe la production d'IP ${ }_{3}$ stimulée par d'autres récepteurs et, finalement, bloque directement l'étape finale d'exocytose [27]. Une protéine $G$ ne contrôle pas simplement un effecteur, mais exécute un programme complexe de transductions. Cette diversité des programmes dépend de la nature, mais aussi de la stœechiométrie entre récepteurs, protéines $G$ et effecteurs. Ceci explique probablement la diversité des modifications physiologiques qu'une hormone ou un neurotransmetteur peut produire selon la cellule considérée. Il faudra mettre en commun toutes les ressources techniques de la biologie moléculaire et cellulaire pour décoder ces programmes de transduction. Eléments clés de la transduction des signaux externes, les protéines $G$ pourraient être à l'origine de nombreuses maladies. Une maladie génétique, le pseudo-hypopàrathyroïdisme, est due à un défaut de $\mathrm{G}_{\mathrm{s}}$ [28]. Un autre défaut de $G_{\mathrm{s}}$ a été mis en évidence dans des tumeurs hypophysaires [29]. $G_{s}$ mutée serait-elle un oncogène?

\section{Summary}

Regulation of ion channels by G. proteins

The $G$ proteins (proteins which bind GTP) are a family of homologous proteins triggering the coupling of various membrane bound receptors to their cellular effectors. A few years ago, it was believed that $G$ proteins only controlled the activity of enzymes which produce or degrade second messengers. Two years ago, it was found that these proteins are also able to couple directly receptors and ionic channels. The best known example is the coupling of muscarinic receptors to $\mathrm{K}^{+}$ channels through a $G$ protein called $G_{k}$. Following these studies, the notion appeared that a given receptor does not trigger its cellular action by modifying a single coupling mechanism, but by executing a complex program of transductions. What will be the next cellular function recognized to be controlled by $\mathrm{G}$ proteins? A role in the traffic of membrane bound proteins is likely. One can also think of a control of cytoskeletal protein functions and, why not, the modulation of gene expression?

\section{TIRÉS A PART}

J. Bockaert. 